The changes in the armed forces medical services merely reflect the changing role of the armed forces themselves and the operational scenarios for which medical support might be required in the future. The fact that the core hospital at Haslar will be for all three services and enlarged in terms of both the number of beds and the range of specialties represented will enable us to provide an even greater range of postgraduate training than at present. The introduction of the military units in large district general hospitals will again allow greater training opportunities than we have previously been able to provide in our small service hospitals. I can therefore confidently state that training opportunities for the doctors who remain will be enhanced and their service caree patterns will not be affected. Uniformed patients will be referred, as at present, to uniformed consultants, either at the core hospital at Haslar or if it is more convenient, to the nearest militarycivilian unit, and they will receive the same treatment as they presently enjoy.

TONY REVELI Ministry of Defence,

London WC1X 8RY

1 Beecham L. Reductions in defence medical services causes BMA concern. BMF 1995;310:196. (21 January.)

\section{Life expectancy in children with cerebral palsy}

EDrTOR,-In their paper on life expectancy in children with cerebral palsy' Jane L Hutton and colleagues remark on our findings in two selected studies, ${ }^{23}$ stating that our subjects with developmental disability seemed to have a much shorter life expectancy than they found. We studied people who had devastating learning disabilities as well as people with less severe disabilities. Some groups had a very poor prognosis-that is, those who were immobile, could not roll over, were tube fed, had no use of their arms or hands, etc. In one of these groups who could roll over, however, estimated survival was similar to that in Hutton and colleagues' group who required a wheelchair.

We did not use the wheelchair item in our papers because it did not discriminate between the degrees of immobility. Hutton and colleagues chose to ignore differences in the severity of disabilities, including levels of mobility and types of feeding, when they discussed the differences between our findings and theirs.

To prove our point we adopted their cruder definitions of mobility-that is, wheelchair required and help needed for propulsion-and a separate category for manual dexterity, defined as inability to feed and dress without help, as well as their years of birth and degree of learning disability (all had cerebral palsy). We used all ages of admission to the California system. We estimated survival over 11 years for 3792 people meeting the wheelchair criteria. The five and 10 year cumulative proportions surviving were almost identical with those in Hutton and colleagues' study. When we used the criterion "does not feed or dress self without help" our cumulative proportions surviving after five and after 10 more years were also similar to those of Hutton and colleagues. We also used period life tables for Hutton and colleagues' definitions of mobility and manual dexterity. We selected subjects who received services during 1987-90 and estimated their average remaining lifetime. The results again were similar to the median survival estimated by Hutton and colleagues. These findings merely confirm that the definitions used by Hutton and colleagues did not have the precision necessary to replicate our findings on who would and would not have a reasonable survival outcome.

Diagnoses such as cerebral palsy and learning disabilities are not determinative predictors in as much as most subjects with these diagnoses are not substantially disabled. The category "patients confined to wheelchairs" is too broad to be a definitive predictor. ${ }^{4}$

\section{RICHARD K EYMAN}

Professor

School of Education

University of California,

Riverside,

CA 9252

USA

HERBERT J GROSSMAN

University of Michigan Hospital Professor of medicine

Ann Arbor,

MI 48109-0800,

USA

THOMAS L CALL

Lanterman Developmental Center,

ROBERT H CHANEY

PO Box 100-R,

Pomona,

CA 91768 ,

USA

1 Hutton JL, Cooke T, Pharoah PO. Life expectancy in children with cerebral palsy. $B M 7$ 1994;309:431-5. (13 August.)

2 Eyman RK, Grossman HJ, Chaney RH, Call TL. The life expectancy of profoundly handicapped people with mental retardation. $N$ Engl f Med 1990;323:584-9.

3 Eyman RK, Grossman HJ, Chaney RH, Call TL. Survival of profoundly disabled people with severe mental retardation. $\mathrm{Am}$ profoundly disabled people wit

4 Eyman RK, Borthwick-Duffy SA, Call TL, White JF. Prediction of mortality in community and institutional settings. Foumal of Mental Deficiency Research 1988;32:203-13.

\section{Drivers with sleep apnoea}

EDrTor,-Ashley Woodcock and Patricia Stone pose the major moral dilemma of how to manage patients with sleep apnoea who hold licences to drive public service or heavy goods vehicles and are reluctant to declare their illness to the Driver and Vehicle Licensing Agency. ${ }^{1}$ They mention the temptation to blackmail such patients into treatment with threats of driving bans. The issue here is not the treatment but the ban. The Driver and Vehicle Licensing Agency recommends that people who hold licences to drive public service or heavy goods vehicles should stop driving if they have sleep apnoea. The patient therefore has an obligation to inform the agency about the condition, which is well known to cause accidents, as Woodcock and Stone point out.

Surely there is no moral dilemma here. Doctors' primary responsibility is to the public at large, not to a person who might cause death or disability to other people. The situation is exactly the same in epilepsy. The recommended practice is to inform patients of their statutory responsibility to tell the Driver and Vehicle Licensing Agency about their condition, to write this in the notes, and if necessary to confirm it with a letter to the patients sent by registered post. The patients' response can be checked by asking them, but also by an inquiry to the agency, at which stage the doctor does not need to provide any clinical information. If patients do not report their condition, however, the doctor should send them a letter telling them that he or she is obliged to inform the licensing authority about their condition and recommending that they should consult a solicitor about the issue.

This sounds legalistic, but the communications can be made in a friendly manner, emphasising encouragement rather than threat and expressing regret at the need to take the ultimate action.

N J LEGG

Royal Postgraduate Medical School, Hammersmith Hospital London W12 0NN

1 Woodcock A, Stone P. Should obesity be treated? BMF 1994; 309:1442. (26 November.)

\section{Driving, glaucoma, and the law}

EDITOR,-Contrary to the impression given by $\mathrm{T}$ Potamitis and colleagues, ${ }^{1}$ glaucoma in a single eye need not be notified to the Driver and Vehicle Licensing Agency. The condition can be regarded as a prospective disability and therefore notifiable as soon as field loss in both eyes occurs. It is the patient's responsibility to notify the agency, but the doctor needs to remind the patient of his or her obligations.

The minimum visual field for safe driving is a field of vision of at least $120^{\circ}$ on the horizontal measured by a Goldmann perimeter with the III4e settings (or equivalent perimetry). In addition, there should be no appreciable field defect in the binocular field that encroaches within $20^{\circ}$ of fixation either above or below the horizontal meridian. By this means homonymous or bitemporal defects that come close to fixation, whether hemianopic or quadrantanopic, are not accepted as safe for driving. Isolated scotomas represented in the binocular field near to the central fixation area may also be inconsistent with safe driving. ${ }^{2}$ Only if the minimum visual field for safe driving cannot be achieved will the driving licence be revoked.

E A ROWSE

Senior medical advise

MARTYN FORD Legal adviser

Driver and Vehicle Licensing Agency,

Swansea SA99 1 TU

1 Potamitis T, Aggarwal RK, Tisaloumas M, Rene C, McLaughlin J, O'Neill E. Driving, glaucoma, and the law. $B M M^{\prime} 1994 ; 309$ : 1057-8. (22 October.)

2 Royal College of Ophthalmologists. Definitions of the minimum field of vision for safe driving for group. Ordinary drivers. London: RCO, 1994.

\section{Making routine data adequate to support clinical audit}

\section{"Routine" is inadequately defined}

EDITOR,-In their editorial on the need for adequate routine data to support clinical audit Martin McKee and colleagues fall into their own trap. ${ }^{1}$ They claim that unambiguous definitions are needed, yet use ambiguous words in their title. Our interpretation of "routine data" is data collected as byproducts of clinical care by the clinician and as an integral part of the process of care. Their definition is that the data are managerial data collected through processes outwith the clinician's control. On the basis of their definition it is not surprising that the data are incomplete and invalid. We have shown that data collected as byproducts of clinical care are valid and able to inform education and service planning, with resultant improvement in the service provided. ${ }^{23}$

It is not surprising that routine data as McKee and colleagues understand the term are inadequate to support audit and contracting. The way ahead for the health service must be to establish a culture in which accurate, structured data collection is an integral part of the process of care, as much second nature to clinicians as it has been to write unstructured notes in the clinical record in the past. When that culture is achieved clinicians will own their own data and trust the information that emerges. Then it will be of use not only for clinical audit but also for activity analysis, service planning, management of resources, and research. JG WILLIAMS

School of Postgraduate Studies in Director

Medical and Health Care,

Morriston Hospital,

Swansea SA6 6NL

1 McKee M, Dixon J, Chenet L. Making routine data adequate to support clinical audit. BMF 1994;309:1246-7. (12 November.) 2 Williams JG, Greenway SC, Morgan JM, Cheung WY. Report of 
a demonstrator project under strand 1 of the integrated clnical workstation (ICWS) programme. Loughborough: Centre for Coding and Classification, 1993.

3 Williams JG, Morgan JM, Greenway SC, Cheung WY. Improving outpatient services: the value of information. In: Ozbolt ing outpatient services: the value of information. In: Ozbolt J 18 th annual symposium on computer applications in medical care, Washington, Nov 1994. Philadelphia: Hanley and Belfus, 1994:978.

\section{Data collection should be integrated with patient care}

EDITOR,-The editorial on making routine data adequate to support clinical audit mentions the high quality of data from the St Mary's maternity information system, ${ }^{1}$ citing two recent papers on its validity. ${ }^{23}$ The editorial's authors, however, have missed the point about why this particular system has proved so successful. The basis of the system is that midwives enter the data themselves and then in turn use the system for practical purposes directly associated with patients' carefor example, it generates summaries and discharge letters. The system is thus integrally associated with patients' care and is not a separate task.

This experience strongly suggests that abandoning the normal practice of relegating the input of data to clerical staff results in better quality of data. This is both because the motivation of health care professionals is higher and, crucially, because of the reinforcing feedback loop involving the use of the information for patients' care.

The Department of Health has introduced an information management and technology strategy for the NHS in England, which has many positive features. ${ }^{4}$ Its potential Achilles' heel, however, is precisely this question of the quality of the data. Although it includes a data quality initiative, this is concerned mainly with administrative data (the postcode and codes for the provider, health authority, and general practitioner), the only clinical element being the code for the diagnosis. We suggest that a great deal could be learnt from the strengths and weaknesses of existing systems, especially successful ones like St Mary's maternity information system, and could then be used in the development of the future information technology for health care.

MICHAEL JOFF

Senior lecture

Academic Department of Public Health St Mary's Hospital Medical School,

London W2 IPG

JEAN CHAPPLE Honorary senior lecturer R W BEARD Professo

Department of Obstetrics and Gynaecology,

St Mary's Hospital Medical School

1 McKee M, Dixon J, Chenet L. Making routine data adequate to

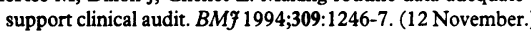

2 Cleary R, Beard RW, Coles J, Devlin HB, Hopkins A, Robert $\mathrm{S}$, et al. The quality of routinely collected maternity data. Bry Obstet Gynaecol (in press).

3 Cleary R, Beard RW, Coles J, Devlin B, Hopkins A, Schumacher $D$, et al. Comparative hospital databases: value for management and quality. Quality in Health Care 1994;3:3-10.

4 Information Management Group of the NHS Managemen Information Management Group of the NHS Managem
Executive. IMET strategy overview. London: HMSO, 1992.

5 Information Management Group of the NHS Management Executive. Setting standards for data quality. London: HMSO, 1993.

\section{Myths in medicine}

EDrToR,-Richard Horton wishes to lay the Jenner myth to rest for the final time. ${ }^{1}$ It was a common belief among farmers in the west country that previous infection with cowpox prevented smallpox. Acting on this, Benjamin Jesty, a farmer in Dorset, infected his wife and two sons with cowpox from a cow. ${ }^{2}$ Twenty years later, on 14 May 1796, Jenner inoculated James Phipps with material from a sore on the hand of Sarah Nelmes, who had been infected with cowpox when milking Blossom.
Jenner thus showed that "true" cowpox could be transmitted from person to person. This was important, since an infected cow was not always available. Jenner then inoculated James Phipps with smallpox two months later and showed that inoculation with cowpox did prevent smallpox. Jenner repeated the experiments. He published his findings in a pamphlet entitled "An inquiry into the causes and effects of the variolae vaccinae ... known by the name of the cow pox" in 1798 . He spent much time answering questions and meeting criticism. He published further pamphlets. $\mathrm{He}$ once described himself as "the vaccine clerk of the world."

It is for this that Jenner received and deserves recognition. The bicentenary of Jenner's experimental proof of the effectiveness of vaccination will be celebrated next year.

C BRUCE PERRY Past chairman, Jenner Trusts IAN BAILEY

Coombe Dingle, Chairman, Jenner Educational Trust

1 Horton R. Myths in medicine. BMF 1995;310:62. (7 January.) 2 Perry CB. Edward fenner. Bristol: University Press, 1983:21.

**Interestingly, the $B M \mathcal{F}$ published the following letter and editorial footnote in October 1895 (BMF 1895;ii:871).

SIR,-During my rambles in Dorsetshire a few weeks ago I noticed an inscription on a tombstone in the little village churchyard of Worth Matravers as follows: "Sacred to the memory of Benj. Jesty Downstay, who departed this life April 16th, 1816. Born at Yetminster in this county. A honest and upright man. He was the first person (known) that introduced cowpox with inoculation, who from great strength of mind made experiment from the cow on his wife and two sons in the year 1774."

I have since ascertained that there is no doubt as to the correctness of this statement, and the above was a farmer in that neighbourhood, and there remains no doubt that he performed vaccination, at least on his own family, several years before Jenner made the discovery.-I am, etc,

Bristol, Sept. 25th

G. C. Pauli, M.R.C.S.

\section{The then editor added the footnote:}

There is no reason to doubt the correctness of the statement made upon the tombstone. It was long a matter of traditional belief amongst the dairy farmers in England and in Holstein that cow-pox was protective against small-pox. The deliberate introduction of cow-pox may therefore well have been adopted by several independent minds. All great discoveries have been anticipated in a similar manner, but it detracts nothing from the greatness of him who first systematises or renders them available for the purposes of every day life.

\section{Sudden infant death syndrome in Maoris}

EdrTor,-Charles Essex's news item on the sudden infant death syndrome in New Zealand quotes the opinion of Dr Ed Mitchell, who coordinated the New Zealand cot death study from 1987 to 1990 and has continued to monitor baby care practices in New Zealand. ${ }^{1}$ Essex points out the disproportionate rate of the syndrome among Maori babies. It must be appreciated, however, that a designated Maori can have any proportion of Maori blood. The New Zealand study called a baby Maori if either parent considered himself or herself to be Maori. But the Maori part was seldom full Maori. For some, it was as little as a quarter or less. Thus the relative rates of the sudden infant death syndrome for Maoris and non-Maoris vary according to the criteria that are used.

The article is accompanied by a photograph of a Maori woman in a flax skirt who is holding a baby and standing in front of the traditional meeting

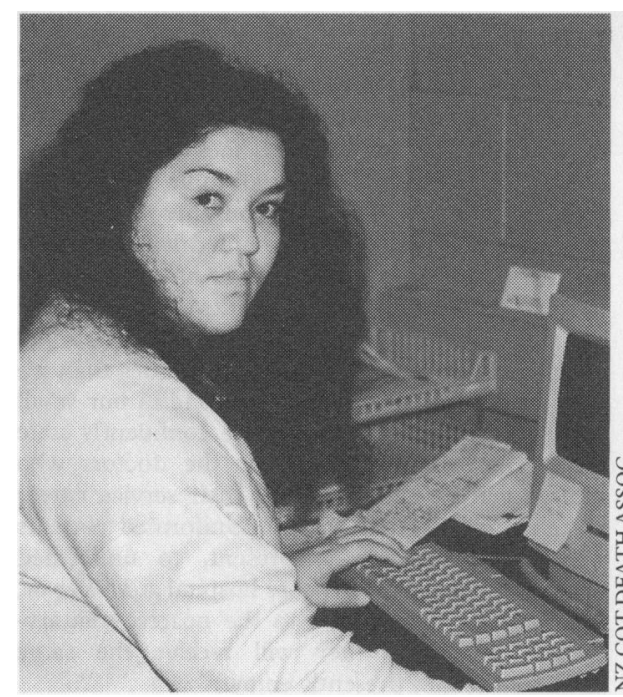

Maoris today live like other New Zealander

house. This gives the impression that this is the manner in which Maoris live in New Zealand. Nothing could be further from the actuality. The situation depicted in the photograph is one used for tourist publicity. Maoris today live like other New Zealanders. Their education is equal, and most of them live in similar houses, enter the same professions and occupations, and are just as familiar with late 20 th century technology (figure).

S L TONKIN

New Zealand Cot Death Association,

National coordinator

PO Box 28177,

Auckland 5, New Zealand

1 Essex C. Cot deaths. New Zealand: campaign falters over bed sharing. BMF 1995;310:8-9. (7 January.)

We apologise for the unintended offence caused in New Zealand by our use of an old picture, and we are grateful to the New Zealand Cot Death Association for supplying us with modern pictures that we can use in the future.-ED, $B M^{\text {\% }}$

\section{n of 1 trials in osteoarthritis}

\section{Study was conceptually and statistically} biased

EDITOR,-Lyn March and colleagues' study comparing a non-steroidal anti-inflammatory drug with paracetamol in osteoarthritis displays conceptual and statistical bias towards paracetamol. ${ }^{1}$ The conceptual bias-that non-steroidal antiinflammatory drugs are illogical when there is little inflammation-would not arise if these drugs were called potent pain killers. After all, paracetamol has antipyretic activity but is used in the absence of fever. Statistical bias arose by the authors' designation of paracetamol as the drug of choice if differences from diclofenac did not reach significance. It was impossible, however, for diclofenac to be significantly better on a global rating: when all preferences were for diclofenac the $P$ value was only 0.06 . In fact, 12 patients showed more preferences for diclofenac (compared with one with more preferences for paracetamol); nine patients had more pain and eight more stiffness when taking paracetamol (compared with one and two, respectively, when taking diclofenac); and 12 needed more escape analgesia when taking paracetamol (compared with one when taking diclofenac (median 7.5 tablets $v 1, P=0 \cdot 13$ )). Eight patients reported more side effects while taking paracetamol compared with five while taking diclofenac; median number six $v$ three, $\mathrm{P}=0 \cdot 17$ ). These results suggest that paracetamol was relatively ineffective and poorly tolerated.

Other studies in osteoarthritis also show that paracetamol is less effective than non-steroidal 